\title{
ПОДХОД К АВТОМАТИЗИРОВАННОМУ ФОРМИРОВАНИЮ НОРМ ВРЕМЕНИ НА ТЕХНИЧЕСКОЕ ОБСЛУЖИВАНИЕ СРЕДСТВ КИПИА, АСУ ТП И СООРУЖЕНИЙ СВЯЗИ ПРЕДПРИЯТИЙ НЕФТЕГАЗОВОЙ ОТРАСЛИ
}

\author{
Савельев Алексей Олегович', \\ sava@tpu.ru
}

\author{
Комагоров Владимир Петрович', \\ komagorov@tpu.ru
}

\author{
Михалевич Сергей Сергеевич', \\ mihalevichss@tpu.ru
' Национальный исследовательский Томский политехнический университет, Россия, 635050, г. Томск, пр. Ленина, 30.

\begin{abstract}
Актуальность исследования обусловлена необходимостью постоянного обновления и пересмотра стратегии повышения общей экономической эффективности предприятий нефтегазовой отрасли путем оптимизации затрат на проведение мероприятий технического обслуживания критически важных контрольно-измерительных приборов и автоматики (КИПиА), оборудования автоматизированных систем управления технологическим процессом (АСУ ТП) и сооружений связи.

Цель: разработать научно обоснованный подход к автоматизированному формированию норм времени на техническое обслуживание оборудования КИПиА, АСУ ТП и сооружений связи предприятий нефтегазодобывающей отрасли, позволяющий сократить время и повысить общую эффективность процесса планирования технического обслуживания.

Объекты: технологические установки добычи нефти и газа, подготовки, перекачки и хранения нефти и газа, поддержания пластового давления, трубопроводного транспорта, контролируемые и управляемые с помощью оборудования АСУ ТП, оснащенные различными приборами КИПиА; сооружения связи: радиорелейные и спутниковые системы связи, проводные системы связи, волоконно-оптические линии связи, телефонные станции, транковые системы связи, системы видеонаблюдения и сигнализации.

Методы. Для автоматизации процесса формирования норм трудозатрат на техническое обслуживание нового оборудования используется метод классификации оборудования по категориям технической сложности.

Результаты. Разработана методика автоматизированного расчета нормативов трудозатрат на техническое обслуживание оборудования предприятий нефтегазовой отрасли, основанная на категориях технической сложности. Определены требования к модулю формирования базовых норм трудозатрат информационной системы управления техническим обслуживанием, выполнена соответствующая программная реализация. На основе предложенной методики сформированы базовые нормы трудозатрат на техническое обслуживание средств АСУ ТП, КИПиА и сооружений связи ОАО «Томскнефть» ВНК.
\end{abstract}

\section{Ключевые слова:}

Система управления техническим обслуживанием, стоимость жизненного цикла,

стратегии технического обслуживания, автоматизация производства, нормирование трудозатрат.

\section{Введение}

Анализ стоимости жизненного цикла производства является критически важным для промышленных предприятий [1-3]. Результаты анализа используются для выбора оптимальной стратегии повышения общей экономической эффективности производства. При этом расходы на реализацию мероприятий технического обслуживания оборудования являются одним из основных элементов, определяющих итоговую стоимость жизненного цикла продукции (рис. 1) [1, 4]. Разработка надежной и грамотной стратегии планирования и проведения мероприятий технического обслуживания позволяет повысить общую экономическую эффективность производственного процесса.

В обобщенном виде все подходы к техническому обслуживанию и ремонту оборудования могут быть сведены к двум основным: проактивному [5-7] и реактивному [2]. Первый заключается в обеспечении максимально возможного межремонтного срока эксплуатации за счет регулярных мероприятий обнаружения и исправления причин отказов оборудования. Реактивный подход, напротив, предполагает ремонт и замену оборудования только в случае полной выработки им своего эксплуатационного ресурса. Иными словами, реактивный подход восстанавливает функциональность технической системы, а проактивный - сохраняет её [2].

В зависимости от особенностей производственного процесса и подхода к управлению формируется оптимальный план мероприятий, как правило, сочетающий реактивные и проактивные подходы [8-10]. С учетом того, что стоимость запасных частей и замены оборудования находятся вне зоны влияния промышленного предприятия, грамотное нормирование труда при выполнении операций технического обслуживания является базовым требованием, от которого зависит общая эффективность процесса планирования.

Для предприятий нефтегазовой отрасли ключевыми категориями оборудования, обеспечиваю- 


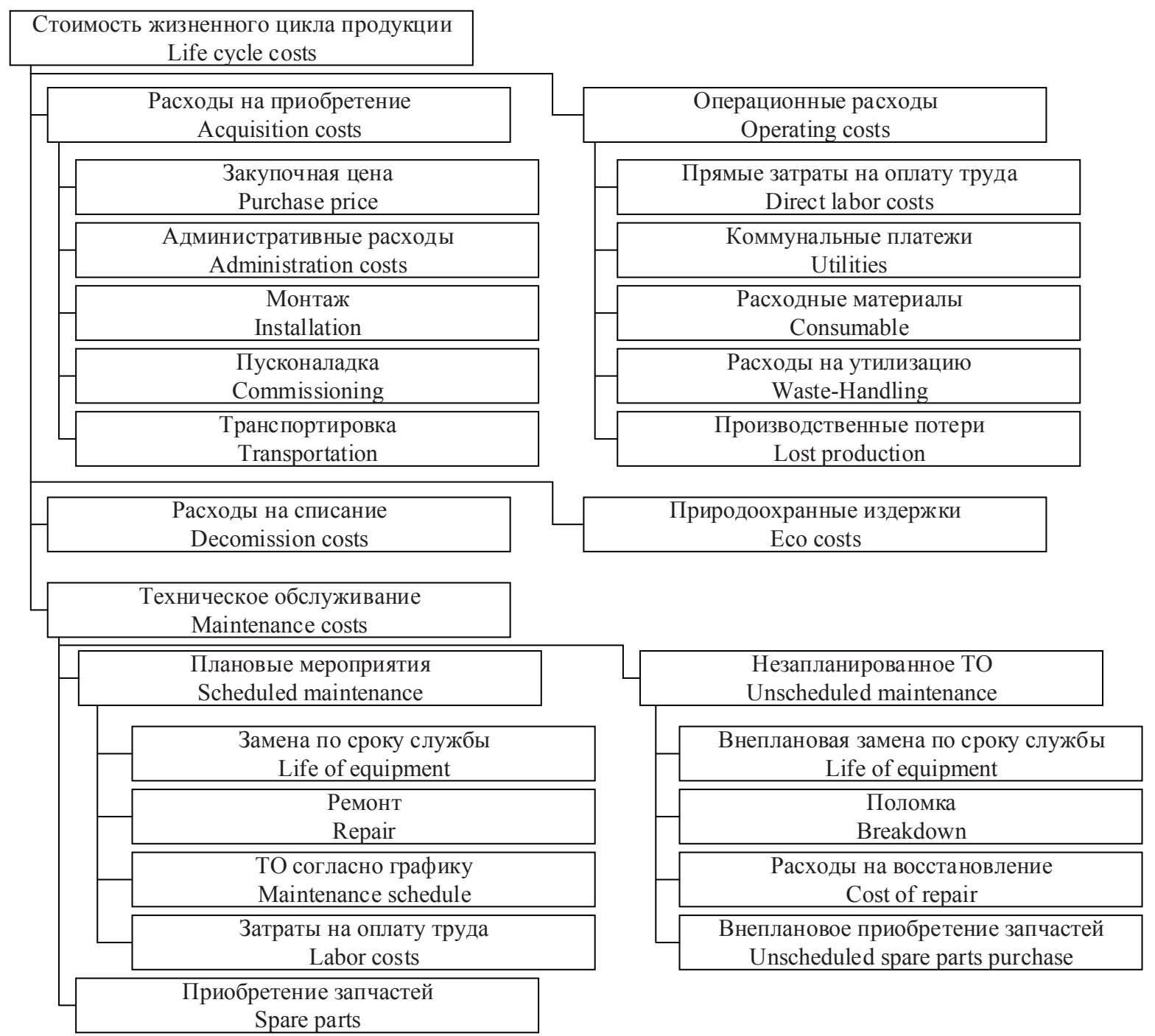

Pис. 1. Основные составляющие стоимости жизненного цикла продукции

Fig. 1. Main components of production life cycle cost

щими возможность оперативного управления производством, являются средства КИПиА, АСУ ТП и сооружения связи. При этом само предприятие представляет собой сложную, разветвленную структуру регионального масштаба, использующую в своей работе широкую номенклатуру оборудования, которое в свою очередь отличается по характеристикам в рамках устройств одной группы и сходного назначения.

Сложившиеся условия значительно ограничивают возможности нормирования труда при техническом обслуживании: метод фотографии рабочего времени в масштабах организации является дорогостоящим и, что более важно, трудоёмким, использование типовых норм затруднено ввиду постоянной модернизации и совершенствования техники. Таким образом, наиболее адекватным в сложившейся ситуации методом является сравнительное нормирование (или нормирование по аналогии).
Таким образом, актуальной является задача разработки методики автоматизированного формирования норм трудозатрат на выполнение операций технического обслуживания оборудования, учитывающей обозначенные особенности эксплуатации средств КИПиА, АСУ ТП и сооружений связи предприятий нефтегазовой отрасли.

\section{Методология исследований}

В рамках разработки подхода к автоматизированному формированию норм времени на техническое обслуживание был проведен анализ следующих основополагающих документов, находящихся в открытом доступе и используемых в нефтегазовой отрасли:

1. Типовые нормы времени на монтаж контрольно-измерительных приборов и средств автоматики в нефтяной промышленности [11].

2. Приказ Министерства здравоохранения и социального развития РФ от 14 октября 2011 г. N 1175н «Об утверждении Межотраслевых ти- 
повых норм времени на работы по сервисному обслуживанию оборудования телемеханики, сопровождению и доработке программного обеспечения» [12].

3. Укрупненные нормы времени на техническое обслуживание, ремонт и метрологическое обеспечение контрольно-измерительных приборов и автоматики (ОАО «Лукойл» НК, Москва 2013 г.) [13].

4. Единые нормы времени на монтаж и демонтаж нефтепромыслового оборудования (утвержденные Министерством нефтяной промышленности 29.09.1983) [14].

5. Доработанный текст проекта Приказа Министерства энергетики РФ «Об утверждении Правил организации технического обслуживания и ремонта оборудования, зданий и сооружений электростанций и сетей» (подготовлен Минэнерго России 08.08.2017) [15].

6. Правила эксплуатации и безопасности обслуживания средств автоматизации, телемеханизации и вычислительной техники в газовой промышленности (утверждены министерством газовой промышленности 03.03.1983) [16].

Также были рассмотрены внутренние регламенты и иные документы, нормирующие операции технического обслуживания, доступ к которым был предоставлен в рамках выполнения научноисследовательских работ по теме «Разработка и исследование прототипа системы расчета и хранения нормативов трудозатрат на техническое обслуживание и ремонт оборудования и сооружений связи ОАО «Томскнефть» ВНК» в 2014-2015 гг.:

1. АСУ ТП и средства КИПиА. Нормы времени на техническое обслуживание (Составитель - ОАО «ВНИИОЭНГ», Москва 2005 г.).

2. Типовые нормы времени на ремонт контрольно-измерительных приборов в нефтяной промышленности (Составитель - ОАО «ВНИИОЭНГ», Москва 2002 г.).

3. Нормы времени на ремонт нефтепромыслового оборудования (Составитель - ОАО «Томскнефть» ВНК, Стрежевой 2013 г.).

4. Техническое обслуживание средств измерения, автоматики, телемеханики и АСУ ТП (Составитель - ПАО «НК «Роснефть», Москва 2007 г.).

5. Техническое обслуживание средств КИПиА. Нормы времени, расхода материалов и транспортных затрат (ОАО «ВНИИОЭНГ»), Москва 2008 г.).

6. Нормы времени на техническое обслуживание, ремонт и метрологическое обеспечение приборов и оборудования систем измерений количества нефти и нефтепродуктов (ОАО «ВНИИОЭНГ»), Москва 2009 г.).

7. АСУ ТП и средства КИПиА. Нормы времени на техническое обслуживание (ОАО «ВНИИОЭНГ»), Москва 2005 г.).

8. Транспортное обслуживание при сервисе. Средства КИПиА и АСУ ТП. Нормы времени (ОАО «ВНИИОЭНГ»), Москва 2007 г.).
9. Типовые нормы времени по техническому обслуживанию средств автоматизации (ОАО «ТомскНИПИнефть ВНК», Томск 2002 г.).

В каждом из рассмотренных источников содержится подробная информация о регламентах проведения технического обслуживания по группам оборудования и типовым нормам времени на выполнение соответствующих операций. Однако существует ряд сложностей, препятствующих использованию имеющихся регламентов при разработке стратегии технического обслуживания оборудования. Во-первых, основой ряда документов являются нормы времени, разработанные и утвержденные более 15 лет назад, соответственно, не учтенными являются особенности современного оборудования. Во-вторых, документы не содержат алгоритма действий по формированию норм времени для новых вводимых в эксплуатацию моделей. Следует отметить, что, несмотря на указанные недостатки, имеющиеся регламенты и нормы могут быть использованы в качестве базовых, отправных значений.

На основе анализа приведенных источников были сформулированы требования к методике автоматизированного расчета нормативов трудозатрат, а также подобраны соответствующие основные положения и расчетные формулы методики.

\section{Требования к методике расчетов нормативов трудозатрат}

Разрабатываемая методика должна обеспечивать не только создание инструмента расчета базовых норм времени на техническое обслуживание оборудования, но и решение следующего ряда дополнительных задач:

1) регламентировать порядок расчета трудозатрат на выполнение технического обслуживания средств АСУ ТП, КИПиА и сооружений связи;

2) формировать единый инструмент расчета стоимости работ для любых объектов АСУ ТП, КИПиА и сооружений связи, с учетом возможности появления новых объектов.

3) разработать дополнительные инструменты оценки работ: нормативного срока выполнения, нормативного количества исполнителей и нормативной продолжительности по каждому из видов работ;

4) спроектировать механизм переутверждения норм и корректирующих коэффициентов.

Решение вышеперечисленных задач потребовало разработки следующих общих положений и допущений:

1. При расчете трудозатрат принимается во внимание то обстоятельство, что продолжительность работ зависит от квалификационного уровня исполнителей.

2. Основой методики и алгоритмов расчета нормативов трудозатрат являются экспертные оценки, сложившиеся в профильных научно-исследовательских институтах. Для уточнения значений предлагается ввести поправочные коэф- 
фициенты, значения которых должны определяться эмпирическим путем.

3. Трудозатраты на выполнение работ рассчитываются по составным блокам, на основе базового классификатора работ.

4. Разработанная методика предназначена для вычисления норм в относительно «простых» случаях, без учета форс-мажорных и иных осложняющих обстоятельств. Для регулировки "сложности» работ (в том числе удаленности объекта, температуры окружающего воздуха и т. п.) предлагается использовать дополнительные поправочные коэффициенты.

\section{Методика автоматизированного} формирования норм трудозатрат

В соответствии с перечнем выявленных требований была разработана методика авторизированного формирования норм трудозатрат на ТО средств КИПиА, АСУ ТП и сооружений связи, включающая:

1) перечень базовых норм, сформированный на основе доступной отраслевой документации [11-17];

2) список категорий технической сложности (КТС) оборудования;

3) алгоритм расчета базовых норм времени для новых моделей вводимого в эксплуатацию оборудования.

В современных условиях техника и технология постоянно меняются, в течение короткого периода времени происходит процесс модернизации, совершенствования существующей техники. Кроме того, в связи с широкой номенклатурой средств КИПиА, идентичных по конструкции, но имеющих некоторые отличия, нормирование всего спектра средств КИПиА является очень трудоемким и сложным процессом, требующим значительных временных затрат. В связи с этим с целью повышения эффективности использования системы нормативов было бы целесообразно использование переводного коэффициента, в качестве которого возможно применение коэффициента сложности технического обслуживания, характеризующего трудоемкость операций при техническом обслуживании средств КИПиА.

Основные принципы использования подобного коэффициента в области автоматизированных систем управления изложены в системе нормативных документов в строительстве ГЭСНп 81-04-02-2001 [17].

Степень сложности обслуживания средств КИПиА может быть оценена категориями технической сложности. Чем сложнее прибор и выше его основные технологические и метрологические характеристики, тем выше его категория технической сложности. Категория технической сложности присваивается каждой единице оборудования. В табл. 1 приведены характеристики технической сложности средств КИПаА для предприятий нефтегазовой отрасли [17].
Однако простое распределение средств КИПиА по категориям технической сложности исказит реально необходимые нормы времени для различных типов приборов, так как каждый тип имеет различную трудоемкость технического обслуживания, связанную с рядом технических особенностей: сложностью монтажа и демонтажа, количеством исполнительных механизмов, сложностью радиоэлементной базы, расширенной функциональностью и т. д. В связи с этим, на основе уже разработанных нормативов, необходимо: 1) распределить средства КИПиА по группам, отражающим функциональное предназначение прибора (сигнализаторы, уровнемеры, регистраторы и т. п.); 2) в каждой созданной группе присвоить средствам КИПиА категории технической сложности согласно табл. 1.

Первая категория технической сложности для каждой созданной группы по аналогии с ГЭСНп 81-04-02-2001 принимается базовой.

Соответственно, $H_{\mathrm{вp}}^{\sigma}=H_{\mathrm{вp}}^{I}$ и $K_{I}=1$, где $H_{\mathrm{вp}}^{\sigma}-$ базовая норма времени на техническое обслуживание средств КИПиА, чел/ч; $H_{\text {вр }}^{I}$ средняя норма времени на техническое обслуживание средств КИПиА I категории технической сложности, которая определяется в чел/ч по формуле:

$$
\dot{I}_{\mathrm{cp}}^{I}=\frac{\sum_{1}^{n} H_{\mathrm{Bp} \_ \text {I }}^{I}}{n},
$$

где $n$ - количество приборов в рассматриваемой группе, которым присвоена I категория технической сложности, шт.; $H_{\text {вр_п }}^{I}$ - норма времени на техническое обслуживание $n$-го прибора I категории технической сложности в рассматриваемой группе, чел/ч.

По имеющимся нормативам произвести расчет средних приближенных коэффициентов $K_{\text {II }}, K_{\text {III }}$ для каждой группы:

$$
\begin{gathered}
K_{I I}=\frac{H_{\mathrm{cp}}^{I I}}{H_{\mathrm{cp}}^{I}}, \\
K_{I I I}=\frac{H_{\mathrm{cp}}^{I I I}}{H_{\mathrm{cp}}^{I}},
\end{gathered}
$$

где $H_{\text {cp }}^{I I}, H_{\text {ср }}^{I I}$ - средние нормы времени на техническое обслуживание средств КИПиА II и III категорий технической сложности, рассчитываемые аналогично формуле (1) с соответствующей корректировкой по категориям технической сложности.

В ходе определения коэффициентов и базовой нормы времени могут возникнуть ситуации, при которых невозможно произвести расчеты по формулам (1)-(3). Способы решения подобных трудностей сведены в табл. 2 .

При внесении информации по нормам времени для нового средства КИПиА используется следующий алгоритм.

1. Новое средство КИПиА добавляется в ранее созданную группу, отражающую его функцио- 
Таблица 1. Категории технической сложности средств КИПиА

Table 1. Technical complexity categories of instrumentation and automation equipment

\begin{tabular}{|c|c|}
\hline \begin{tabular}{|c} 
Категория техни- \\
ческой сложности \\
Technical \\
complexity category
\end{tabular} & $\begin{array}{c}\text { Характеристика оборудования } \\
\text { Equipment characteristics }\end{array}$ \\
\hline$K_{I}$ & $\begin{array}{l}\text { Измерительные, регулирующие, преобразующие, передающие на дальние расстояния устройства, электромагнитные, по- } \\
\text { лупроводниковые и другие компоненты, сигнальная арматура и т. п. приборного или аппаратного типов исполнения, ха- } \\
\text { рактеризующиеся: } \\
\text { · простым контролем, измерением параметров состояния технологического объекта; } \\
\text { · одноконтурным автоматическим регулированием; } \\
\text { · автоматическим однотактным логическим управлением (переключения, блокировки и т. п.); } \\
\text { · отсутствием предварительной обработки информации; } \\
\text { · классом точности ниже или равным } 1,0 \\
\text { Measuring, regulating, transforming, long distances transmitting devices, electromagnetic, semiconductor and other com- } \\
\text { ponents characterized by: } \\
\text { · simple control, technological object parameters measurement; } \\
\text { · single-loop automatic regulation; } \\
\text { · automatic single-ended logic control (switching, blocking, etc.); } \\
\text { · lack of information preprocessing; } \\
\text { - accuracy class lower than or equal to } 1,0\end{array}$ \\
\hline$K_{I I}$ & 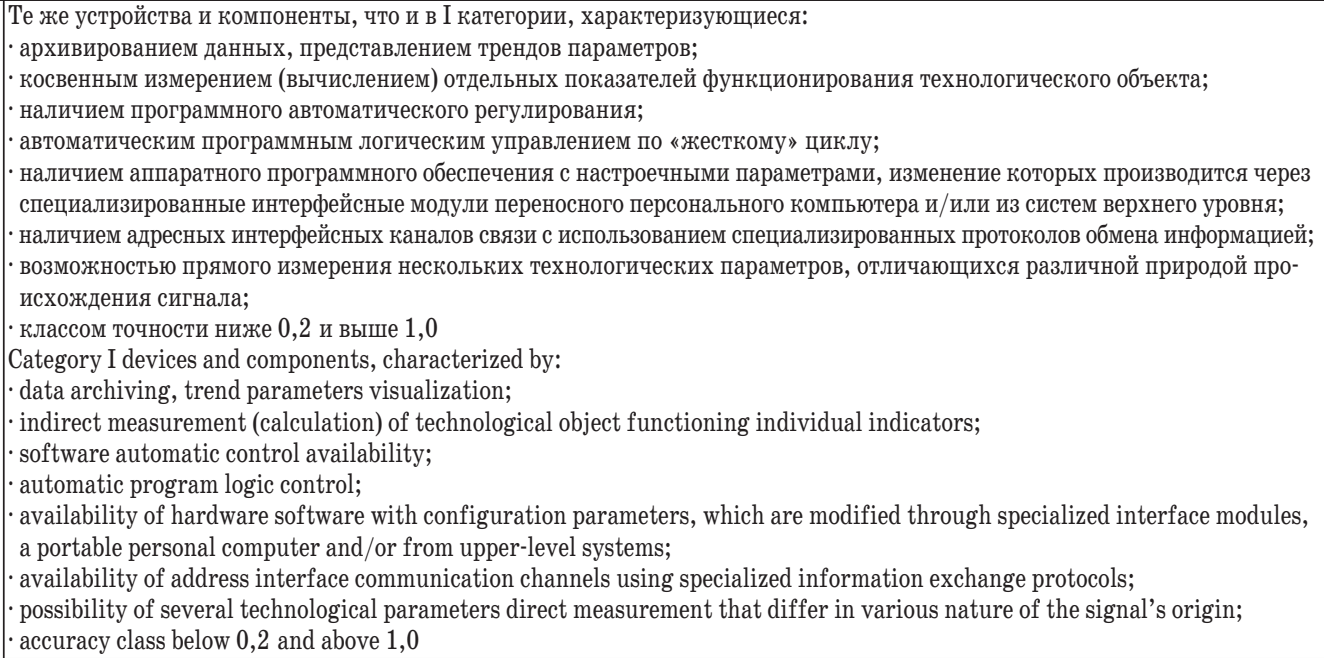 \\
\hline$K_{I I I}$ & $\begin{array}{l}\text { Те же устройства и компоненты, что и во II категории, а также закрытые автоматизированные системы управления тех- } \\
\text { нологическими процессами, предварительно программируемые «интеллектуальные» логические контроллеры (PLC), } \\
\text { массовые расходомеры, характеризующиеся: } \\
\text { · возможностью анализа и оценки состояния процесса по его модели (распознавание ситуации, диагностика аварийных } \\
\text { состояний); } \\
\text { · возможностью управления быстропротекающими процессами в аварийных условиях или управления с адаптацией (сам- } \\
\text { ообучением и изменением алгоритмов и параметров); } \\
\text { · возможностью оптимального управления установившимися режимами (в статике) и/или переходными процессами (оп- } \\
\text { тимизация в динамике); } \\
\text { классом точности выше или равным 0,2 } \\
\text { The same devices and components as in Category II as well as the closed automated control systems of pre-programmed intelli- } \\
\text { gent logic controllers, mass flow meters, characterized by: } \\
\text { · ability to analyze and assess the state of the process according to its model (situation recognition, emergency conditions diag- } \\
\text { nostics); } \\
\text { - ability to control fast processes in emergency conditions or management with adaptation (self-learning and changing algo- } \\
\text { rithms and parameters); } \\
\text { - possibility of the steady-state regimes (static) and/or transient processes (optimization in dynamics) optimal control; } \\
\text { - accuracy class higher than or equal to } 0,2\end{array}$ \\
\hline
\end{tabular}

нальное предназначение. При отсутствии таковой создается новая группа.

2. Устройству присваивается КТС методом экспертной оценки или в соответствии с ГЭСНп 81-04-02-2001.

3. Рассчитывают норму времени на техническое обслуживание прибора в зависимости от присвоенной категории технической сложности по следующим формулам:

$$
H_{\mathrm{Bp}}^{I}=H_{\mathrm{Bp}}^{\sigma} ;
$$

$$
\begin{aligned}
& H_{\mathrm{вp}}^{I I}=H_{\mathrm{вp}}^{\sigma} \cdot K_{I I} ; \\
& H_{\mathrm{вp}}^{I I I}=H_{\mathrm{вp}}^{\sigma} \cdot K_{I I I},
\end{aligned}
$$

где $H_{\mathrm{вp}}^{I}, H_{\mathrm{вp}}^{I I}, H_{\mathrm{вp}}^{I I I}$ - нормы времени на техническое обслуживание средств КИПиА I, II и III категорий технической сложности соответственно, чел/ч.

В случае отсутствия в рассматриваемой группе базовой нормы времени $H_{\text {вр }}^{\circ}$ производить расчет по следующим формулам: 
Таблица 2. Способы решения трудностей при расчете КТС

Table 2. Solutions in calculating the technical complexity category

\begin{tabular}{|c|c|}
\hline Возникшие трудности при расчетах/Difficulties in calculations & Способ решения/Solution \\
\hline $\begin{array}{l}\text { В рассматриваемой группе отсутствуют средства КИПиА, относящиеся к І категории } \\
\text { технической сложности } \\
\text { In the group there are no control and testing instrumentations related to the first catego- } \\
\text { ry of technical complexity }\end{array}$ & $\begin{array}{l}K_{I I}, K_{\text {III }}, H_{\mathrm{cp}}^{I} \text { не рассчитываются. } \\
\text { Необходимо произвести расчет только } H_{\mathrm{cp}}^{I I} \text { и } H_{\mathrm{cp}}^{I I} \\
\text { The } K_{I I}, K_{I I I} \text { and } H_{\mathrm{cp}}^{I} \text { coefficients are not calculated. } \\
\text { Only } H_{\mathrm{cp}}^{I I} \text { and } H_{\mathrm{cp}}^{I I I} \text { should be calculated }\end{array}$ \\
\hline $\begin{array}{l}\text { В рассматриваемой группе отсутствуют средства КИПиА, относящиеся ко II катего- } \\
\text { рии технической сложности } \\
\text { In the group there are no control and testing instrumentations related to the second cate- } \\
\text { gory of technical complexity }\end{array}$ & $\begin{array}{l}\text { Коэффициент } K_{I I} \text { не рассчитывается } \\
\text { The } K_{I I} \text { coefficient is not calculated }\end{array}$ \\
\hline $\begin{array}{l}\text { В рассматриваемой группе отсутствуют средства КИПиА, относящиеся к III катего- } \\
\text { рии технической сложности } \\
\text { In the group there are no control and testing instrumentations related to the third cate- } \\
\text { gory of technical complexity }\end{array}$ & $\begin{array}{l}\text { Коэффициент } K_{I I I} \text { не рассчитывается } \\
\text { The } K_{I I I} \text { coefficient is not calculated }\end{array}$ \\
\hline $\begin{array}{l}\text { В рассматриваемой группе отсутствуют средства КИПиА, относящиеся ко II и III ка- } \\
\text { тегорий технической сложности } \\
\text { In the group there are no control and testing instrumentations related to the second and } \\
\text { third category of technical complexity }\end{array}$ & $\begin{array}{l}\text { Принять значения коэффициентов, согласно ГЭСНп } \\
\text { 81-04-02-2001: } K_{I I}=1,313 ж K_{I I I}=1,566 \\
\text { The following coefficients must be accepted in accordance } \\
\text { with GESNp 81-04-02-2001: } K_{I I}=1,313 ж K_{I I I}=1,566\end{array}$ \\
\hline
\end{tabular}

$$
\begin{gathered}
H_{\mathrm{вp}}^{I I}=H_{\mathrm{cp}}^{I I} ; \\
H_{\mathrm{вp}}^{I I I}=H_{\mathrm{cp}}^{I I I} .
\end{gathered}
$$

1. При невозможности применения формул (4)-(7) или (8) для нового средства КИПиА формулируются соответствующие обоснования. В этом случае будет рассмотрена необходимость определения нормы времени на техническое обслуживание данных средств КИПиА методом проведения фотографии рабочего дня.

2. Для средств КИПиА, имеющих в своем составе расширяемые каналы формирования входных и выходных сигналов, производить расчет норм времени на техническое обслуживание в зависимости от фактического использования данных каналов (например, к такому устройству относится контроллер).

Под каналом формирования входных и выходных сигналов (каналов) следует понимать совокупность технических средств и линий связи, обеспечивающих преобразование, обработку и передачу информации для использования в системе АСУ ТП. При этом учитывается количество каналов:

- информационных (в том числе каналов измерения, контроля, оповещения, адресных, состояния и т. п.);

- управления;

- в составе информационных каналов и каналов управления в свою очередь учитывается количество каналов:

а) дискретных - контактные и бесконтактные на переменном и постоянном токе, импульсные от дискретных (сигнализирующих) измерительных преобразователей, предназначенные для контроля состояния различных двухпозиционных устройств, а также для передачи сигналов типа «включить-выключить» и т. п.;

б) аналоговых, к которым относятся все остальные - токовые, напряжения, частоты, взаимной индуктивности, естественные или унифицированные сигналы измерительных пре- образователей (датчиков), которые изменяются непрерывно, кодированные (импульсные или цифровые) сигналы для обмена информацией между различными цифровыми устройствами обработки информации и т. п.

Расчет нормы времени на техническое обслуживание средства КИПиА с расширяемым количеством каналов [17]:

$$
\begin{gathered}
H_{\mathrm{вр}}^{*}=H_{\mathrm{вр}}+ \\
+\frac{H_{\mathrm{u}}^{\text {д }} \cdot K_{\mathrm{u}}^{\text {Д }}+H_{\mathrm{u}}^{\mathrm{a}} \cdot K_{\mathrm{u}}^{\mathrm{a}}+H_{\mathrm{y}}^{\text {д }} \cdot K_{\mathrm{y}}^{\text {म }}+H_{\mathrm{y}}^{\mathrm{a}} \cdot K_{\mathrm{y}}^{\mathrm{a}}}{60},
\end{gathered}
$$

где $H_{\mathrm{вp}}^{*}$ - норма времени на техническое обслуживание устройства с учетом фактически используемых каналов формирования входных и выходных сигналов, чел/ч; $H_{\text {вр }}$ - базовая норма времени на техническое обслуживание устройства без учета имеющихся каналов формирования входных и выходных сигналов, чел/ч.

Условные обозначения и значения остальных параметров формулы (9) приведены в табл. 3, 4 [17].

\begin{tabular}{|c|c|c|}
\hline $\begin{array}{l}\text { Условное } \\
\text { обозначение } \\
\text { Symbol }\end{array}$ & $\begin{array}{c}\text { Наименование } \\
\text { Name }\end{array}$ & $\begin{array}{l}\text { Ед. изм. } \\
\text { Unit of mea- } \\
\text { surement }\end{array}$ \\
\hline$K_{\mathrm{n}}^{\mathrm{a}}$ & $\begin{array}{l}\text { Количество фактически используемых } \\
\text { информационных аналоговых каналов } \\
\text { The number of actually used analogue in- } \\
\text { formation channels }\end{array}$ & \multirow{4}{*}{$\begin{array}{l}\text { шт. } \\
\text { piece }\end{array}$} \\
\hline$K_{\mathrm{u}}^{\mathrm{B}}$ & $\begin{array}{l}\text { Количество фактически используемых } \\
\text { информационных дискретных каналов } \\
\text { The number of actually used discrete in- } \\
\text { formation channels }\end{array}$ & \\
\hline$K_{\mathrm{y}}^{\mathrm{a}}$ & $\begin{array}{l}\text { Количество фактически используемых } \\
\text { аналоговых каналов управления } \\
\text { The number of actually used analog con- } \\
\text { trol channels }\end{array}$ & \\
\hline$K_{\mathrm{y}}^{\nexists}$ & $\begin{array}{l}\text { Количество фактически используемых } \\
\text { дискретных каналов управления } \\
\text { The number of actually used discrete con- } \\
\text { trol channels }\end{array}$ & \\
\hline
\end{tabular}

Таблица 3. Условные обозначения каналов передачи информации и управления

Table 3. Symbols of information transmission and control channels 
Таблица 4. Условные обозначения и значения норл трудозат рат на ТО каналов передачи инфорлации и управле ния

Table 4. Legend and values of labor costs time norms for informa tion transfer and control channels maintenance

\begin{tabular}{|c|c|c|c|}
\hline $\begin{array}{c}\text { Условное } \\
\text { обозначение } \\
\text { Symbol }\end{array}$ & $\begin{array}{c}\text { Наименование } \\
\text { Name }\end{array}$ & $\begin{array}{c}\text { Значение } \\
\text { Value }\end{array}$ & \begin{tabular}{|l|} 
Ед. изм. \\
Unit of mea- \\
surement
\end{tabular} \\
\hline$H_{\text {й }}^{\mathrm{a}}$ & $\begin{array}{l}\text { Норма времени трудовых зат- } \\
\text { рат на ТО одного информацион- } \\
\text { ного аналогового канала } \\
\text { The time rate of labor costs for } \\
\text { one information analog channel } \\
\text { maintenance }\end{array}$ & 1,37 & \multirow{4}{*}{$\begin{array}{c}\text { чел/мин } \\
\text { man-minute }\end{array}$} \\
\hline$H_{\mathrm{H}}^{\text {म }}$ & $\begin{array}{l}\text { Норма времени трудовых зат- } \\
\text { рат на ТО одного информацион- } \\
\text { ного дискретного канала } \\
\text { The time rate of labor costs for } \\
\text { one discrete information channel } \\
\text { maintenance }\end{array}$ & 0,67 & \\
\hline$H_{\mathrm{y}}^{\mathrm{a}}$ & $\begin{array}{l}\text { Норма времени трудовых зат- } \\
\text { рат на ТО одного аналогового } \\
\text { канала управления } \\
\text { The time rate of labor costs for } \\
\text { one analog control channel main- } \\
\text { tenance }\end{array}$ & 7,47 & \\
\hline$H_{\mathrm{y}}^{\mathrm{A}}$ & $\begin{array}{l}\text { Норма времени трудовых зат- } \\
\text { рат на ТО одного дискретного } \\
\text { канала управления } \\
\text { The time rate of labor costs for } \\
\text { one discrete control channel ma- } \\
\text { intenance }\end{array}$ & 1,31 & \\
\hline
\end{tabular}

\section{Программное обеспечение автоматизированного формирования норм трудозатрат}

Предложенная методика автоматизированного формирования норм трудозатрат реализована в виде соответствующего алгоритмического и программного обеспечения, основанного на трехуровневой архитектуре: сервер базы данных, сервер приложений (web-сервер) и клиент (браузер). Были спроектированы и реализованы следующие интерфейсы для:

1) работы с устройствами КИПиА;

2) работы с устройствами АСУ ТП;
3) работы с устройствами сооружений связи;

4) просмотра регламентов технического обслуживания;

5) работы с данными. В интерфейс входят следующие подинтерфейсы: а) интерфейс добавления новых устройств в базу данных; б) интерфейс редактирование существующих устройств в базе данных; в) интерфейс удаления устройств из базы данных; г) интерфейс для работы с регламентами ТО: привязка к устройствам, удаление работ у устройства.

Фрагмент интерфейса для работы с устройствами АСУ ТП представлен на рис. 2.

На рис. 3 представлена еЕРС диаграмма (событийная цепочка процессов), описывающая в обобщенном виде последовательность действий пользователя при вводе в эксплуатацию новой модели оборудования. Результатом является добавление норм времени на ТО в соответствующие разделы базы данных (БД) программного обеспечения автоматизированного формирования нормативов трудозатрат.

Для эффективного применения алгоритма расчета базовых норм для новых устройств была разработана подробная классификация средств КИПиА, АСУ ТП и сооружений связи для предприятий нефтегазовой отрасли, содержащая более 500 групп устройств, распределенных по 16 базовым категориям.

Применение разработанной методики и основанного на ней программного обеспечения автоматизированного формирования нормативов трудозатрат обеспечивает информационную поддержку следующих связанных с планированием мероприятий технического обслуживания задач управления [18-20].

1) формирование графиков планово-предупредительного ремонта и проверки исправности средств КИПиА, АСУ ТП и сооружений связи;

2) формирование отчетов по количеству средств КИПиА, АСУ ТП и сооружений связи, используемых в рамках производственного процесса;

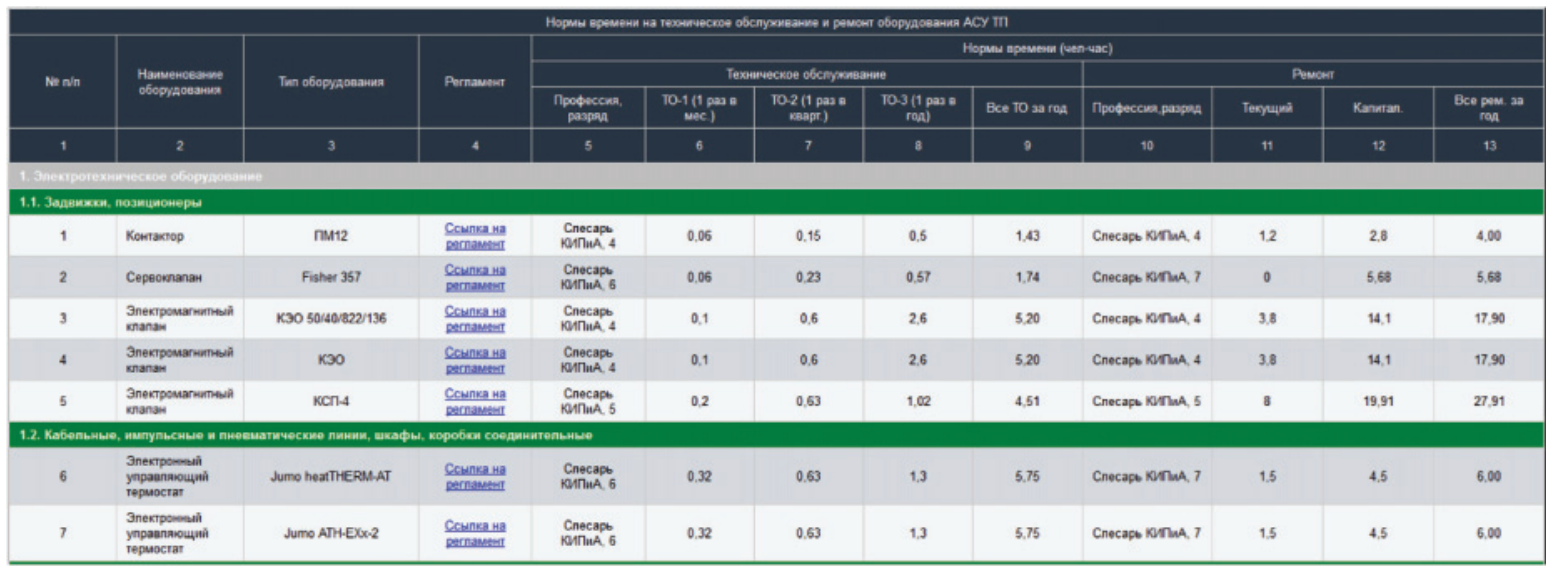

Pис. 2. Фрагмент интерфейса для работы с устройствали АСУ ТП

Fig. 2. Fragment of the APCS equipment software interface 


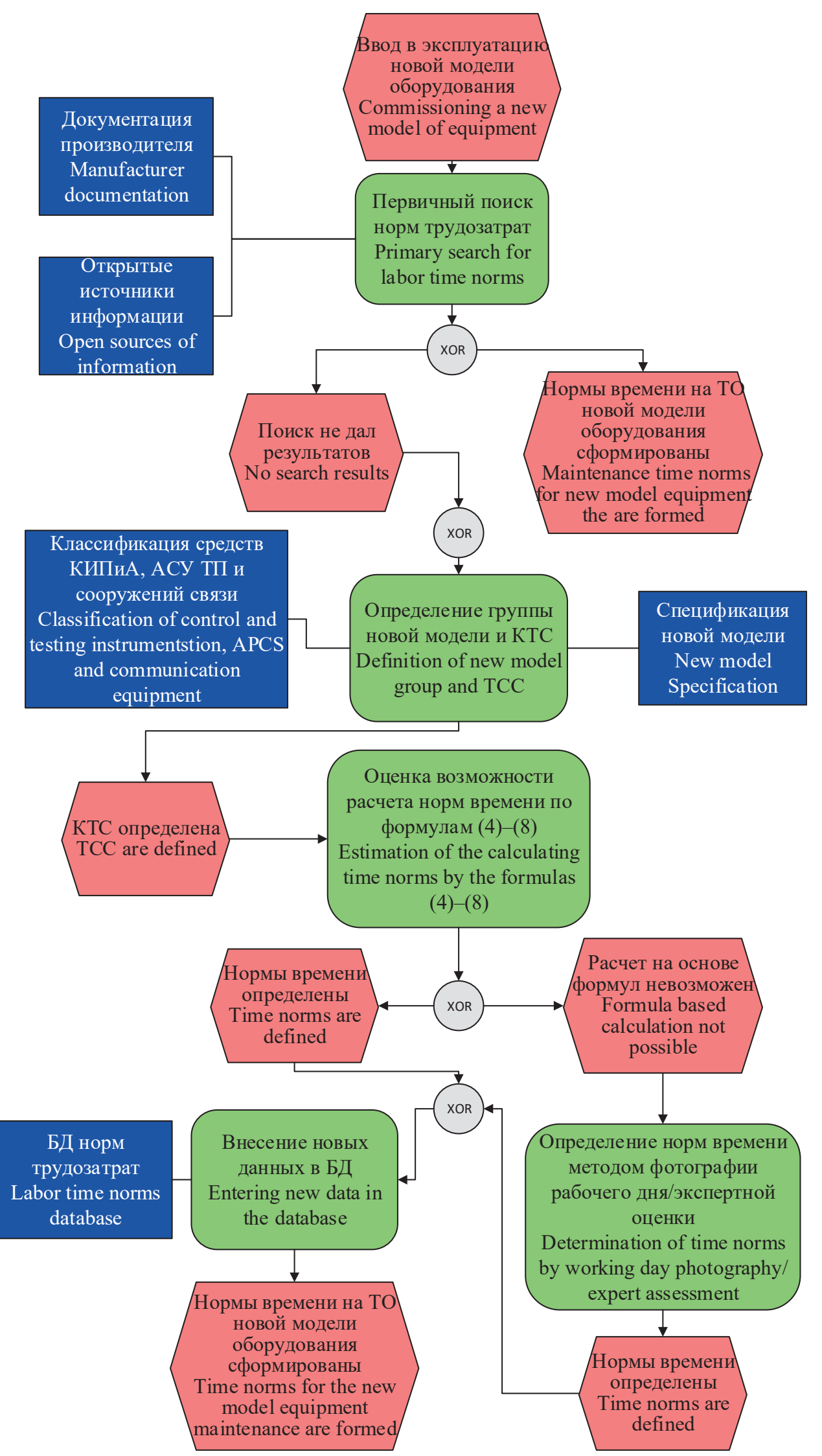

Pис. 3. Событийная цепочка проиессов формирования норм времени на ТО новой модели оборудования

Fig. 3. eEPC-diagram of maintenance time norms generation for a new equipment model 
3) расчет времени (человеко-часов) на проведение ТО имеющихся объемов средств КИПиА, АСУ ТП и сооружений связи.

По вопросам, связанным с приобретением и адаптацией разработанного решения, можно обратиться в отделение информационных технологий Национального исследовательского Томского политехнического университета, или по e-mail авторов статьи.

\section{Заключение}

В ходе проведенного исследования была разработана методика автоматизированного формирования базовых норм трудозатрат на проведение операций технического обслуживания средств КИПиА, АСУ ТП и сооружений связи для предприятий нефтегазовой отрасли. Методика обеспечивает единый регламент порядков расчета норм трудозатрат и возможность формирования норм для новых единиц оборудования, а также обладает возможностью адаптации для применения в рамках промышленных предприятий других отраслей народного хозяйства.

\section{СПИСОК ЛИТЕРАТУРЫ}

1. Maintenance decision support for manufacturing systems based on the minimization of the life cycle cost / A. Reina, A. Kocsis, A. Merlo, I. Nemeth, F. Aggogeri $/ / 49^{\text {th }}$ CIRP Conference on Manufacturing Systems. - Germany, 2016. - P. 674-679.

2. Silivant D. Reliability centered maintenance cost modeling: Lost opportunity cost // Annual Reliability and Maintainability Symposium (RAMS). - Palm Harbor, USA, 2015. - article № 7105111 .

3. Verma N.K., Subramanian T.S.S. Cost benefit analysis of intelligent condition based maintenance of rotating machinery $/ / 7^{\text {th }}$ IEEE Conference on Industrial Electronics and Applications (ICIEA). Singapore, 2012. URL: https://ieeexplore.ieee.org/stamp/ stamp.jsp?tp=\&arnumber=6360940 (дата обращения 09.10.2018

4. Chan T., de Vericourt F., Besbes 0. Contracting in Medical Equipment Maintenance Services: an Empirical Investigation. URL: https://papers.ssrn.com/sol3/papers.cfm?abstract_id=2494265 (дата обращения 09.10.2018).

5. Mushiri T., Hungwe R., Mbohwa C. An artificial intelligence based model for implementation in the petroleum storage industry to optimize maintenance // IEEE International Conference on Industrial Engineering and Engineering Management (IEEM). Singapore, 2017. - P. 1485-1489.

6. Sensors: The Enablers for Proactive Maintenance in the Real World / M. Albano, L.L. Ferreira, G. Di Orio, P. Malo et al. // $5^{\text {th }}$ International Conference on Control, Decision and Information Technologies (CoDIT). - Thessaloniki, Greece, 2018. P. 569-574.

7. Cheng Z., Rong L., Liu Z. A RCM analytical method considering proactive maintenance // International Conference on Quality, Reliability, Risk, Maintenance, and Safety Engineering (QR2MSE). - Chengdu, China, 2013. - P. 1473-1476.

8. Liao W., Wang T. An optimization approach using in production scheduling with different order $/ / 7^{\text {th }}$ International Conference on Industrial Technology and Management (ICITM). - 0xford, UK, 2018. - P. 237-241.

9. Impact of intelligent wireless sensor network on predictive maintenance cost / S. Sadiki, M. Faccio, M. Ramadany, D. Amgouz, S. Boutahar $/ / 4^{\text {th }}$ International Conference on Optimization and
Применение разработанной методики, помимо упорядочивания процесса нормирования трудозатрат, обеспечивает повышение эффективности процессов планирования мероприятий технического обслуживания, что в свою очередь позволяет сформировать более точную оценку стоимости жизненного цикла продукции.

Методика была апробирована при выполнении научно-исследовательской работы по теме «Разработка и исследование прототипа системы расчета и хранения нормативов трудозатрат на техническое обслуживание и ремонт оборудования и сооружений связи ОАО «Томскнефть» ВНК» и позволила в автоматизированном режиме сформировать базовые нормы трудозатрат на техническое обслуживание для более чем 4000 моделей оборудования.

Работа выполнена при финансовой поддержке Минобрнауки РФ, в ралках реализации государственного задания в сфере научной деятельности, выполнения проектов для получения первичных научных результатов, обеспечивающих расширение участия подведолственных образовательных организаций в реализации Национальной технологической инициативы, № 8.10283.2018/11.12.

Applications (ICOA). - Mohammedia, Morocco, 2018. URL: https://ieeexplore.ieee.org/stamp/stamp.jsp?tp=\&arnumber $=8370573$ (дата обращения 09.10.2018).

10. Maintenance scheduling in the electricity industry: a literature review / A. Froger, M. Gendreau, J. Mendoza, E. Pinson, L.M. Rousseau // European Journal of Operational Research. 2016. - V. 251. - № 3. - P. 695-706.

11. Типовые нормы времени на монтаж контрольно-измерительных приборов и средств автоматики в нефтяной промышленности (утв. Приказом Миннефтепрома СССР от 29.09.1987 N 692). URL: http://www.libussr.ru/doc_ussr/usr_14308.htm (дата обращения 09.10.2018).

12. Приказ Министерства здравоохранения и социального развития РФ от 14 октября 2011 г. N 1175н «0б утверждении Межотраслевых типовых норм времени на работы по сервисному обслуживанию оборудования телемеханики, сопровождению и доработке программного обеспечения». URL: http://ivo.garant.ru/\#/document/55172593/paragraph/1:0 (дата обращения 09.10.2018).

13. Укрупненные нормы времени на техническое обслуживание, ремонт и метрологическое обеспечение контрольно-измерительных приборов и автоматики. URL: https://docplayer.ru/ 31837689-Ukrupnennye-normy-vremeni-na-tehnicheskoe-obsluzhivanie-remont-i-metrologicheskoe-obespechenie-kontrolnoizmeritelnyh-priborov-i-avtomatiki.html (дата обращения 09.10.2018).

14. Единые нормы времени на монтаж и демонтаж нефтепромыслового оборудования. URL: http://docs.cntd.ru/document/ 1200082881 (дата обращения 09.10.2018).

15. Доработанный текст проекта Приказа Министерства энергетики РФ «0б утверждении Правил организации технического обслуживания и ремонта оборудования, зданий и сооружений электростанций и сетей” (подготовлен Минэнерго России 08.08.2017). URL: http://base.garant.ru/56725433/ (дата обращения 09.10.2018).

16. Правила эксплуатации и безопасности обслуживания средств автоматизации, телемеханизации и вычислительной техники в газовой промышленности. URL: http://docs.cntd.ru/document/1200003260 (дата обращения 09.10.2018). 
17. ГЭСНп 81-04-02-2001 «Государственные элементные сметные нормы на пусконаладочные работы. Сборник 2. Автоматизированные системы управления (издание 2008 г. с учетом изменений и дополнений)». URL: http://www.norm-load.ru/ $\mathrm{SNiP} /$ Data1/55/55022/index.htm (дата обращения 09.10.2018).

18. Кошкин А.И., Попов Е.А. Разработка информационной системы учета технического обслуживания систем КИПиА, АСУТП и метрологии // Информационные технологии в управлении и экономике. - 2017. - Т 2. - Вып. 7. - С. 45-57.

19. Mainkar M.S., Rathod R.K. Maintenance Management System Effective Tool to Progress and Reach Total Productive Maintenance \& Six Sigma Business Development Strategies // Internatio- nal Conference on Industrial Engineering, Management Science and Application (ICIMSA). - Seoul, South Korea, 2017. URL: https://ieeexplore.iee.org/stamp/stamp.jsp?tp=\&arnumber=7985593 (дата обращения 09.10.2018).

20. Rastegari A., Mobin M. Maintenance decision making, supported by computerized maintenance management system // Annual Reliability and Maintainability Symposium (RAMS). - Tucson, USA, 2016. URL: https://ieeexplore.ieee.org/stamp/stamp.jsp?tp=\&arnumber=7448086 7985593 (дата обращения 09.10.2018).

Поступила 10.10.2018 2.

\section{Информация об авторах}

Савельев A.O., кандидат технических наук, доцент отделения информационных технологий Инженерной школы информационных технологий и робототехники Национального исследовательского Томского политехнического университета.

Комагоров В.П., кандидат технических наук, доцент отделения информационных технологий Инженерной школы информационных технологий и робототехники Национального исследовательского Томского политехнического университета.

Михалевич C.C., кандидат технических наук, научный сотрудник научно-образовательной лаборатории промышленной робототехники Национального исследовательского Томского политехнического университета. 


\title{
APPROACH TO AUTOMATED FORMATION TIME NORMS FOR MAINTENANCE OF CONTROL AND TESTING INSTRUMENTATION, APCS AND COMMUNICATION EQUIPMENT OF OIL AND GAS ENTERPRISES
}

\author{
Aleksey 0. Savelev', \\ sava@tpu.ru \\ Vladimir P. Komagorov', \\ komagorov@tpu.ru \\ Sergey S. Mikhalevich', \\ mihalevichss@tpu.ru \\ ' National Research Tomsk Polytechnic University, \\ 30, Lenin Avenue, Tomsk, 634050, Russia.
}

The relevance of the research is caused by the need to update and revise constantly the strategy of oil and gas enterprises economic efficiency by optimizing the costs of control and testing instrumentation, APCS and communication equipment maintenance.

The main aim of the research is to develop a scientifically based approach to the automated maintenance time standard of control and testing instrumentation, APCS and communication equipment. The approach should allow reducing the time and increasing the overall efficiency of the maintenance planning process.

Objects of the research are technological installations of oil and gas production, preparation, pumping and storage, reservoir pressure maintenance; transport pipeline, equipment for automated process control systems; communication facilities: radio-relay and satellite communication systems, wired communication systems, fiber-optic communication lines, telephone exchanges, trunk communication systems, video surveillance and alarm systems.

Methods. To automate the formation of labor standards for maintenance of new equipment, the method of classifying equipment into categories of technical complexity was used.

Results. The authors have developed the automated calculation method for maintenance of labor cost standards. The method is based on the categories of technical complexity. The requirements for formation of software basic labor norms were determined. On the basis of the proposed methodology, basic labor standards for control and testing instrumentation, APCS and communication equipment maintenance were formed (within Tomskneft company).

\section{Key words:}

Maintenance management system, life cycle cost, maintenance strategies, production automation, labor costing.

The research was financially supported by the Ministry of Education and Science of the RF within the implementation of the State Task in the field of scientific activities, projects for obtaining primary scientific results ensuring the extend of participation of subordinate educational establishments in implementation of the National Technological Initiative, no. 8.10283.2018/11.12.

\section{REFERENCES}

1. Reina A., Kocsis A., Merlo A., Nemeth I., Aggogeri F. Maintenance decision support for manufacturing systems based on the minimization of the life cycle cost. $49^{\text {th }}$ CIRP Conference on Manufacturing Systems. Germany, 2016. pp. 674-679.

2. Silivant D. Reliability centered maintenance cost modeling: Lost opportunity cost. Annual Reliability and Maintainability Symposium (RAMS). Palm Harbor, USA, 2015. Article № 7105111.

3. Verma N.K., Subramanian T.S.S. Cost benefit analysis of intelligent condition based maintenance of rotating machinery. $7^{\text {th }}$ IEEE Conference on Industrial Electronics and Applications (ICIEA). Singapore, 2012. Available at: https://ieeexplore.ieee.org/stamp/stamp.jsp?tp=\&arnumber $=6360940$ (accessed 09 October 2018).

4. Chan T., De Vericourt F., Besbes 0. Contracting in Medical Equipment Maintenance Services: an Empirical Investigation. Available at: https://papers.ssrn.com/sol3/papers.cfm?abstract id $=2494265$ (accessed 09 October 2018).

5. Mushiri T., Hungwe R., Mbohwa C. An artificial intelligence based model for implementation in the petroleum storage industry to optimize maintenance. IEEE International Conference on Industrial Engineering and Engineering Management (IEEM). Singapore, 2017. pp. 1485-1489.
6. Albano M., Ferreira L.L., Di Orio G., Malo. P. etc. Sensors: The Enablers for Proactive Maintenance in the Real World. $5^{\text {th }}$ International Conference on Control, Decision and Information Technologies (CoDIT). Thessaloniki, Greece, 2018. pp. 569-574.

7. Cheng Z., Rong L., Liu Z. A RCM analytical method considering proactive maintenance. International Conference on Quality, Reliability, Risk, Maintenance, and Safety Engineering (QR2MSE). Chengdu, China, 2013. pp.1473-1476.

8. Liao W., Wang T. An optimization approach using in production scheduling with different order. $7^{\text {th }}$ International Conference on Industrial Technology and Management (ICITM). 0xford, UK, 2018. pp. 237-241.

9. Sadiki S., Faccio M., Ramadany M., Amgouz D., Boutahar S. Impact of intelligent wireless sensor network on predictive maintenance cost. $4^{\text {th }}$ International Conference on Optimization and Applications (ICOA). Mohammedia, Morocco, 2018. Available at: https://ieeexplore.ieee.org/stamp/stamp.jsp?tp=\&arnumber $=8370573$ (accessed 09 0ctober 2018).

10. Froger A., Gendreau M., Mendoza J., Pinson E., Rousseau L.M. Maintenance scheduling in the electricity industry: a literature review. European Journal of Operational Research, 2016, vol. 251 , no. 3, pp. 695-706. 
11. Tipovye normy vremeni na montazh kontrolno-izmeritelnykh priborov $i$ sredstv avtomatiki $v$ neftyanoy promyshlennosti [Typical standard time for installation of instrumentation and automation in the oil industry]. Available at: http://www.libussr.ru/doc ussr/usr_14308.htm (accessed 09 October 2018).

12. Prikaz Ministerstva zdravookhraneniya $i$ sotsialnogo razvitiya RF ot 14 oktyabrya 2011 g. $N 1175 n$ [Order of the Ministry of Health and Social Development of the Russian Federation of October 14, $2011 \mathrm{~N} 1175 \mathrm{n}]$. Available at: http://ivo.garant.ru/\#/document/55172593/paragraph/1:0 (accessed 09 0ctober 2018).

13. Ukrupnennye normy vremeni na tekhnicheskoe obsluzhivanie, remont $i$ metrologicheskoe obespechenie kontrolno-izmeritelnykh priborov $i$ avtomatiki [Extended time standards for maintenance, repair and metrological support of instrumentation and automation]. Available at: https://docplayer.ru/31837689-Ukrupnennyenormy-vremeni-na-tehnicheskoe-obsluzhivanie-remont-i-metrologicheskoe-obespechenie-kontrolno-izmeritelnyh-priborov-i-avtomatiki.html (accessed 09 0ctober 2018).

14. Edinye normy vremeni na montazh i demontazh neftepromyslovogo oborudovaniya [Uniform time standards for installation and dismantling of oilfield equipment]. Available at: http://docs. cntd.ru/document/1200082881 (accessed 09 0ctober 2018).

15. Dorabotanny tekst proekta Prikaza Ministerstva energetiki RF «Ob utverzhdenii Pravil organizatsii tekhnicheskogo obsluzhivaniya i remonta oborudovaniya, zdaniy i sooruzheniy elektrostantsiy $i$ setey» [The revised text of the draft Order of the Ministry of Energy of the Russian Federation «On Approval of the Rules for Organizing Maintenance and Repair of Equipment, Buildings and Structures of Power Plants and Networks»]. Available at: http://base.garant.ru/56725433/ (accessed 09 October 2018).

16. Pravila ekspluatatsii i bezopasnosti obsluzhivaniya sredstv avtomatizatsii, telemekhanizatsii $i$ vychislitelnoy tekhniki $v$ gazovoy promyshlennosti [Rules of operation and safety of automation equipment, telemechanization and computing equipment maintenance in the gas industry]. Available at: http://docs.cntd.ru/ document/1200003260 (accessed 09 October 2018).

17. GEHSNp 81-04-02-2001 "Gosudarstvennye elementnye smetnye normy na puskonaladochnye raboty. Sbornik 2. Avtomatizirovannye sistemy upravleniya (izdanie 2008 g. s uchetom izmeneniy i dopolneniy)» [GESNp 81-04-02-2001 «State elemental estimated norms for commissioning and commissioning. Collection 2. Automated control systems (2008 edition with changes and additions)»]. Available at: http://www.norm-load.ru/SNiP/Data1/55/55022/index.htm (accessed 09 0ctober 2018).

18. Koshkin A.I., Popov E.A. Development of an information system for accounting of instrumentation and automation systems, process control systems and metrology maintenance. Information technology in management and economics, 2017, vol. 2, Iss. 7, pp. 45-57. In Rus.

19. Mainkar M.S., Rathod R.K. Maintenance Management System Effective Tool to Progress and Reach Total Productive Maintenance \& Six Sigma Business Development Strategies. International Conference on Industrial Engineering, Management Science and Application (ICIMSA). Seoul, South Korea, 2017. Available at: https://ieeexplore.ieee.org/stamp/stamp.jsp?tp=\&arnumber=7985593 (accessed 09 October 2018).

20. Rastegari A., Mobin M. Maintenance decision making, supported by computerized maintenance management system. Annual Reliability and Maintainability Symposium (RAMS). Tucson, USA, 2016. Available at: https://ieeexplore.ieee.org/stamp/stamp. jsp?tp=\&arnumber $=74480867985593$ (accessed 09 October 2018).

Received: 10 October 2018.

\section{Information about the authors}

Aleksey O. Savelev, Cand. Sc., associate professor, National Research Tomsk Polytechnic University.

Vladimir P. Komagorov, Cand. Sc., associate professor, National Research Tomsk Polytechnic University.

Sergey S. Mikhalevich, Cand. Sc., researcher, National Research Tomsk Polytechnic University. 